\title{
GLOMERULAR CAPILLARY GROWTH AND CELLULAR HYPERPLASIA IN A MODEL OF FOCAL AND SEGMENTAL GLOMERULOSCLEROSIS
}

\author{
JOHN F BERTRAM $^{1}$ AND MEROE M CAHILL ${ }^{2}$ \\ ${ }^{1}$ Department of Anatomy, Monash University, Clayton, Victoria, 3800, ${ }^{2}$ Department of Anatomy and Cell \\ Biology, University of Melbourne, Parkville, Victoria 3052 Australia \\ (Accepted March 16, 2000)
}

\begin{abstract}
Focal and segmental glomerulosclerosis (FSGS) is a chronic renal disorder characterized by segmental glomerular lesions and widespread podocyte foot process effacement. We have previously shown that glomerular enlargement (hypertrophy) precedes the development of FSGS in an animal model not previously thought to involve glomerular hypertrophy. This hypertrophy involved growth of glomerular capillaries. The aim of the present study was to determine whether the capillary growth involved an increase in the number of capillaries per glomerulus, or lengthening of existing capillaries. In addition, we examined the contribution of glomerular cell hyperplasia to the hypertrophy. We found that glomerular capillary growth in this model appears to primarily involve lengthening of existing capillaries rather that sprouting of new capillaries, and that glomerular cell proliferation contributes to the glomerular hypertrophy.
\end{abstract}

Keywords: capillary, focal and segmental glomerulosclerosis, glomerulus, kidney, puromycin aminonucleoside, stereology.

\section{INTRODUCTION}

Focal and segmental glomerulosclerosis (FSGS) is a chronic renal disorder characterized by segmental glomerular lesions and widespread podocyte (glomerular epithelial cell) foot process effacement. Although the aetiology of FSGS is unknown, studies examining FSGS in humans and animal models have demonstrated a link between glomerular hypertrophy (abnormal enlargement of the glomerulus and/or renal corpuscle) and FSGS.

One animal model of FSGS involves multiple administrations of puromycin aminonucleoside (PAN) to rats (Grond et al., 1985; Marinides et al., 1987, 1990). Until our previous study (Cahill et al., 1996) this model was not thought to involve glomerular enlargement. However, we found that mean glomerular volume in PAN-treated rats was almost $50 \%$ greater than in saline-treated rats at week 7 (Cahill et al., 1996). FSGS had not developed at this time. At week 13, mean glomerular volume was more than $60 \%$ greater in PAN-treated rats and FSGS was still minimal. We then determined glomerular capillary growth in these rats. Unbiased stereological methods were used to estimate total capillary length and surface area in average glomeruli. The results indicated biphasic glomerular hypertrophy in this model. In the first phase (0-7 weeks) the glomerular hypertrophy involved an increase in the total length of capillaries per average glomerulus, but during the second phase (7-13 weeks) capillary growth did not appear to contribute to the continued glomerular growth.

The first aim of the present study was to determine whether the lengthening of glomerular capillaries in the PAN-treated rats in the first phase involved lengthening of existing capillaries and/or formation of new capillaries. The number of capillary loops per renal corpuscle was estimated in the same rats studied by Cahill et al. (1996) using the method of Nyengaard and Marcussen (1993). Then, the contribution of glomerular cell hyperplasia to the glomerular hypertrophy in these rats was determined. Here, the optical disector was used to estimate the total number of cells per glomerulus. Finally, PCNA immunohistochemistry was used to assess glomerular cell proliferation in renal corpuscles from a new group of saline and PAN-treated rats.

\section{MATERIALS AND METHODS}

Two groups of adult female Sprague-Dawley rats weighing approximately $200 \mathrm{~g}$ were used in this study. The first group was previously used in Cahill et al. (1996). These rats received injections of either 
normal saline or puromycin aminonucleoside $(2 \mathrm{mg}$ $/ 100 \mathrm{~g}$ bodyweight) at weeks $0,1,2,4,6,8$ and 10 . At weeks 7 and 13, the kidneys were perfusion-fixed with $4 \%$ paraformaldehyde in $0.1 \mathrm{M}$ phosphate buffer for 5 minutes at a pressure of $180 \mathrm{~mm} \mathrm{Hg}$ and processed for stereological analysis. In brief, $1 \mathrm{~mm}$ slices were embedded in glycolmethacrylate (for stereological estimation of glomerular number, mean glomerular volume and in the present study for estimation of the numerical density of glomerular cells) and randomly sampled $1 \mathrm{~mm}^{3}$ pieces of cortex were processed for embedding in epon-araldite (for stereological estimation of glomerular capillary length, surface area, and in the present study, number). For full details of these methods see Bertram et al. (1992), Bertram (1995) and Cahill et al. (1996).

The number of capillary loops per average glomerulus was determined using the method of Nyengaard and Marcussen (1993). In brief, five randomly selected blocks were used from each kidney. The blocks were processed for embedding in eponaraldite. A series of six serial $0.5 \mu \mathrm{m}$ sections was cut from each block, thus ensuring a complete series of three adjacent sections.

Three adjacent $0.5 \mu \mathrm{m}$ sections stained with methylene blue were used from each block with the middle section used as a reference. Two glomerular profiles were selected from each block using the modulus sampling method of Nyengaard (1993a). The area of the two sampled renal corpuscle profiles in the middle sections was estimated using point counting at a magnification of approximately 1,500x. All capillary luminal profiles were identified. The previous adjacent section was then placed on a second matching projection microscope and the capillary luminal profiles compared. Three types of capillary events were counted: luminal connections, luminal fragments and luminal lagoons. Capillary luminal events were counted using physical disectors and capillary number estimated according to Nyengaard and Marcussen (1993).

The numerical density of cells in glomeruli was estimated using optical disectors as previously described (Bertram et al., 1992). In brief, the $20 \mu \mathrm{m}$ haematoxylin and eosin stained glycolmethacrylate sections used in our earlier study in which we estimated glomerular size (Cahill et al., 1996) were analyzed. Glomerular cell number was estimated in four saline-treated and four PAN-treated rats at weeks 7 and 13. Approximately 20 glomeruli were analyzed per rat. Glomerular cell numerical density was estimated as described in Bertram et al. (1992). Then cell numerical density was multiplied by mean glomerular volume to provide an estimate of cell number per average glomerulus.

A second group of rats was used for PCNA immunohistochemistry studies on glomerular cell proliferation. The number of PCNA-positive nuclear profiles per unit area of glomerular profile was estimated. In brief, at weeks 1, 2, 4, 7 and 13 kidneys from two saline-treated rats and three PAN-treated rats were perfused with Hank's balanced salt solution and then the right kidneys were cut into $2 \mathrm{~mm}$ slices and immersion-fixed in Bouin's fixative for 2 hours. The tissue was processed for embedding in paraffin and $3 \mu \mathrm{m}$ sections were cut. PCNA immunohistochemistry was then used to identify dividing cells. Sections were counterstained with haematoxylin. Approximately 70-80 renal corpuscle profiles from three sections per rat were used to count PCNA-positive cells. A mechanized stage was used to systematically sample fields and glomeruli through each section.

Data were analysed using a two-way analysis of variance using Minitab Statistical Software (Minitab Inc., State College, PA, USA). A probability of 0.05 or less was accepted as statistically significant. Values are mean \pm standard deviation.

\section{RESULTS}

The number of capillary loops per glomerulus in saline and PAN-treated rats at weeks 7 and 13 was similar. No difference was found between saline $(132.8 \pm 50.9$ capillary loops per glomerulus at 7 weeks, 6 rats; $142.1 \pm 49.6$ loops at 13 weeks, 6 rats) and PAN-treated $(113.9 \pm 6.6$ loops at 7 weeks, 5 rats; $138.9 \pm 40.4$ loops at 13 weeks, 5 rats) rats.

The total number of cells per glomerulus was significantly greater in PAN-treated rats than salinetreated rats at 7 weeks $(\mathrm{p}<0.05)$, but remained relatively unchanged between 7 and 13 weeks. Values for saline and PAN-treated rats at 7 weeks were $871 \pm 226$ and $1,228 \pm 337$ cells, respectively. At 13 weeks, values for total glomerular cell number in saline and PAN-treated rats were 1,064 \pm 39 and $1,222 \pm 205$, respectively.

At week 1 there was a similar number of PCNApositive cells per renal corpuscle profile in PANtreated rats as in saline-treated rats (see Fig. 1). However, at week 2, renal corpuscle profiles from PAN-treated rats contained more than twice as many PCNA-positive nuclei as did renal corpuscles from saline-treated rats. By weeks 4 and 7, the number of 
PCNA-positive cells per renal corpuscle profile was similar in the two groups. At 13 weeks, one of the PAN-treated rats showed a large increase in the number of PCNA-positive glomerular cells. Based on cell position and nuclear size and shape, these cells could be easily identified as podocytes. The other PAN-treated rats at week 13 showed no increase in the number of PCNA-positive cells within glomeruli.

\section{DISCUSSION}

These results, when considered together with the findings of Cahill et al. (1996) suggest that glomerular capillary growth in this model of FSGS primarily involves lengthening of existing capillaries rather that sprouting of new capillaries. In addition, glomerular cell proliferation appears to contribute to the glomerular hypertrophy.

The finding regarding glomerular capillary growth is somewhat surprising given that the total length of capillaries per average glomerulus in PANtreated rats was $3.97 \mathrm{~mm}$ greater than in salinetreated rats at 7 weeks, and $2.14 \mathrm{~mm}$ greater at 13 weeks. Yet, no increase in the number of capillary loops per renal corpuscle was observed in the PAN-treated rats. Similarly, no evidence of capillary spouting was observed using scanning electron microscopy (data not presented). These results suggest that the increase in the length of capillaries per renal corpuscle in PAN-treated rats is primarily due to lengthening of existing capillaries, not sprouting of new capillary loops. Interestingly, all previous studies that have used the technique of Nyengaard and Marcussen (1993) to estimate the number of capillary loops per glomerulus have found that increases in the number of capillary loops contributed to the increase in total capillary length. These studies include studies of glomerular capillary growth in rats with lithium nephropathy (Marcussen et al., 1994), experimental diabetic nephropathy (Nyengaard and Rasch, 1993) and following unilateral nephrectomy (Nyengaard, 1993b).

It should be stated that the relatively high variance of the estimates of capillary number could be masking true changes in the number of capillaries in the saline and PAN-treated rats. Some of this variance arises from the many steps involved in obtaining the estimates. There is also natural variance between rats, and between renal corpuscles within kidneys. There is also variance within renal corpuscles due to the nature of the Euler-Poincaré characteristic. In the present study, 10 renal corpuscles were used from each rat to estimate capillary number per renal corpuscle in order to minimise the variance due to the level of sectioning through the renal corpuscle.

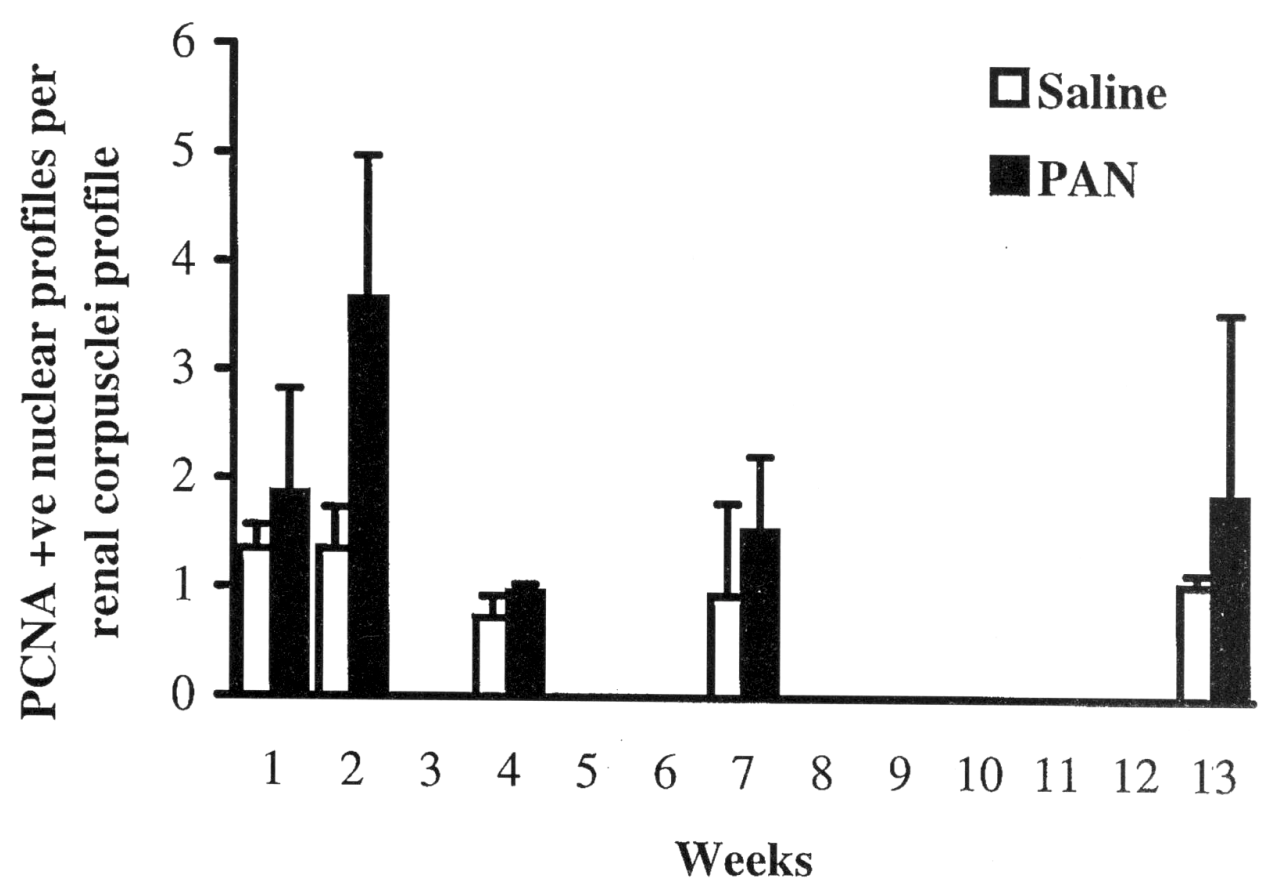

Fig. 1. Histogram showing the number of PCNA-positive cells per renal corpuscle profile in saline and PANtreated rats. 
The present results suggest that cell proliferation contributes to the glomerular hypertrophy with total cell number greater in glomeruli from PAN-treated rats at 7 weeks. Of course this increase in cell number could be due to an increase in the number of resident glomerular cells and/or infiltration of cells. PCNA immunohistochemistry showed that at least some cells in glomeruli were undergoing mitosis. This was particularly the case in PAN-treated rats at weeks 1 and 2. A mixed population of cells appeared to be PCNA-positive and it is not possible without doublelabelling immunohistochemistry to determine whether it is resident and/or infiltrated cells that are dividing. However, at week 13 it was clear that some podocytes in PAN-treated rats were attempting to divide.

In conclusion, the present results indicate that glomerular capillary growth in this PAN model of FSGS involves lengthening of existing capillaries as well as cell proliferation. Further studies are required to identify the molecules that stimulate capillary growth and cell hyperplasia in this model. Once the molecular mechanisms underlying this growth are identified, we will be in a better position to develop strategies for preventing and/or reversing FSGS in animal models, and ultimately in humans.

A preliminary report of some of the data was presented at the $X^{\text {th }}$ International Congress for Stereology, Melbourne, Australia, 1-4 November 1999.

\section{REFERENCES}

Bertram JF (1995). Analyzing renal glomeruli with the new stereology. Int Rev Cytol 161:111-72.
Bertram JF, Soosaipillai MC, Ricardo SD, Ryan GB (1992). Total numbers of glomeruli and individual glomerular cell types in the normal rat kidney. Cell Tissue Res 270:37-45.

Cahill MM, Ryan GB, Bertram JF (1996). Biphasic glomerular hypertrophy in rats administered puromycin aminonucleoside. Kidney Int 50:768-75.

Grond J, Koudstaal J, Elema JD (1985). Mesangial function and glomerular sclerosis in rats with aminonucleoside nephrosis. Kidney Int 27:405-10.

Marcussen N, Nyengaard JR, Christensen S (1994). Compensatory growth of glomeruli is accomplished by an increased number of glomerular capillaries. Lab Invest 70:868-74.

Marinides GN, Groggel GC, Cohen AH, Cook T, Baranowski RL, Westenfelder C, Border WA (1987). Failure of angiotensin converting enzyme inhibition to affect the course of chronic puromycin aminonucleoside nephropathy. Am J Pathol 129:394-401.

Marinides GN, Groggel GC, Cohen AH, Border WA (1990). Enalapril and low protein reverse chronic puromycin aminonucleoside nephropathy. Kidney Int 37:749-57.

Nyengaard JR (1993a). The quantitative development of glomerular capillaries in rats with special reference to unbiased stereological estimates of their number and sizes. Microvasc Res 45:243-61.

Nyengaard JR (1993b). Number and dimensions of rat glomerular capillaries in normal development and after nephrectomy. Kidney Int 43:1049-57.

Nyengaard JR, Marcussen N (1993). The number of glomerular capillaries estimated by an unbiased and efficient stereological method. J Microsc 171:27-37.

Nyengaard JR, Rasch R (1993). The impact of experimental diabetes mellitus in rats on glomerular capillary number and sizes. Diabetologia 36:189-94. 